\title{
Ray-Tracing and 3-D Objects Representation in the BCC and FCC Grids
}

\author{
Luis Ibáñez, Chafiaâ Hamitouche, Christian Roux \\ Département Image et Traitement de l'Information \\ ENST - Bretagne, Technopôle de Brest-Iroise \\ BP 832, 29285 Brest, France \\ E-mail: Luis.Ibanez@enst-bretagne.fr
}

\begin{abstract}
This paper describes a ray-tracing and an object description method for objects sampled not in the usual cubic grid, but in BCC (Body Centered Cubic) and FCC (Face Centered Cubic) grids, which are well known in crystallography. The use of this kind of grids is motivated by their interesting characteristics: they reduce the density of samples needed to represent a signal without information loss and they have better topological properties than the cubic grid.
\end{abstract}

keywords: Image Representation, Rendering, Visualization

\section{Introduction}

Sampling a signal in $3-D$ space satisfying the Shannon theorem leads to the use of the BCC grid as the optimal sampling grid [1]. Its reciprocal, the FCC grid, shows also a good performance from the point of view of low sample density. These grids have more symmetries than the cubic one, which gives a better approach to an anisotropic data representation. For this reason they are recommended as optimal grids for Mathematical Morphology processing [3]. The use of these grids simplifies the surface extraction process in volume data [14]. Recently it has been shown that the use of the BCC and FCC grids enhances the performance of reconstruction from projections methods [10], which leads to the emergence of volume data in BCC and FCC format in 3-D imaging for medical applications.

In spite of their interesting characteristics, the use of these grids is usually rejected because of the increment in algorithmic complexity. This argument is not always true, and it reflects only the lack of deep work in the analysis of the discrete topology corresponding to these grids. The method presented here shows that it is possible to exploit the potential offered by the BCC and FCC grids.

\section{Ray Tracing in Volume Data}

Direct Volume Rendering methods include a ray tracing process in order to compute the contribution of the voxels in the volume to each pixel in the synthesized 


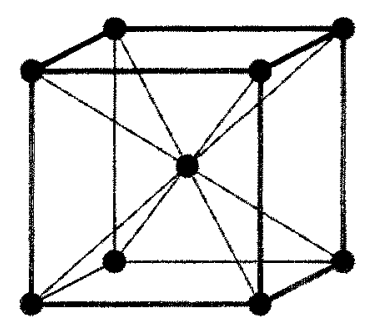

BCC (Body Centered Cubic) Grid

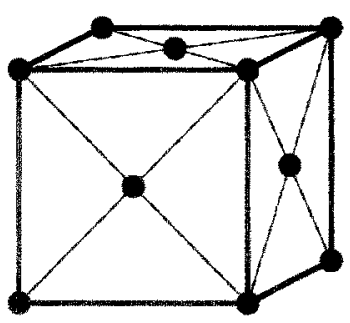

FCC (Face Centered Cubic) Grid

Fig. 1. BCC and FCO grids

image $[9][6][5][7]$. When objects are represented by their discrete surfaces, topological problems arise in the cubic grid, which leads to the possibility of passing a ray through a surface without touching it [4]. To avoid this problem, thicker surfaces or thicker rays should be used. However thicker rays implies a longer processing time in the ray tracing process.

$\mathrm{BCC}$ and $\mathrm{FCC}$ grids do not lead to such topological problems, and allows in a more robust way the notions of discrete curves, surfaces and objects to be defined. Here, the objects are represented on a $\mathrm{BCC}$ or FCC grid by using only their external surfaces. A discrete straight line tracing algorithm has been developed to guide the ray tracing process from each pixel towards the objects in the volume data. Interactions between the ray and the objects surfaces are defined in a discrete way too.

\section{Topology of the BCC and FCC grids}

Topology is defined here in function of the Voronoi cells of each point in the grid. The $3-D$ shape associated to the roxel is the solid polyhedron corresponding to the Voronoi cell (see Figure 3). A connected set of voxels is considered as a $3-D$ Manifold. The Voronoi cells can fill the space by a simple tiling, and the volume data can be considered here as region of the $3-D$ space completely filled with polyhedral voxels.

Definition 1. Two n-dimensional regions of the space are connected if they have a $(n-1)$-dimensional region in common. Two voxels are connected if they have a common face. Two faces are connected if they have a common edge. Two edges are connected if they have a common vertex. The existence of $m$-dimensional regions $(m<(n-1))$ in common between two $n$-dimensional regions does not implies connectivity.

Definition 2. An Object is a set of connected voxels. An Object Surface is the set of faces between object voxels and non-object voxels.

The Voronoi cell in the BCC grid is the Truncated Octahedron, and in the FCC grid is the Rhombic Dodecahedron [2]. BCC and FCC grids have the nice 
property that an edge in the tiling structure is common to exactly three voxels, and hence are common to exactly three faces. When these voxels are classified as belonging to an object or not, always two out of the three voxels belongs to the same class. Then two out of the three faces belong to the object surface. This local property implies that every face in the object surface has exactly one face connected to each of its edges. Then object surfaces are always closed surfaces.

\section{Object Representation}

Volume data represents a $3-D$ signal which has been discretized to be processed. Often the original source of the signal is a continuous real process (as is the case in medical imaging). A classification process should be done in order to determine the regions corresponding to an object. Classification is hard to perform close to the object boundary regions, which leads to a higher classification error for such voxels and therefore to errors in the shape of the external object surface.

When the description of the object surface is limited to the determination of faces belonging or not to the surface, certain information about the real form of the object boundary is lost, and hence the original form cannot be retrieved exactly. This is specially important for processes that must use the surface normal. To avoid this problem a certain amount of information should be left from the classification process to indicate the relative position of a voxel in the transition region.

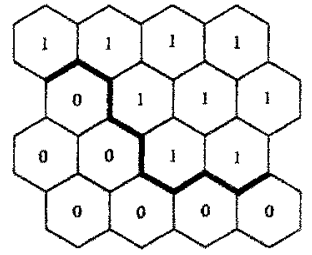

Objex strface

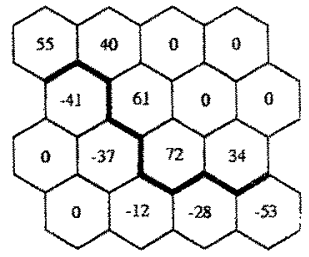

Subwpixel displacernent of $\$$

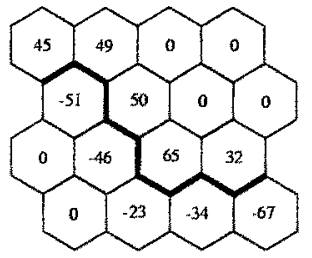

Smooth objeci surface; $\$$

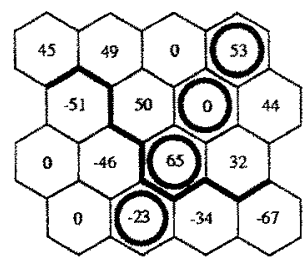

Ray-Surface interaction

Fig. 2. Illustration of smooth curve representation

Definition 3. A Smooth Object Surface is the set of all the voxels in contact with a face of the Object Surface, along with a signed integer coefficient assigned 
to each voxel. The coefficient represents the relative position of the voxel in the transition region. This coefficients take values from -128 to 127 .

Objects represented in this way allow the information of the complete volume data to be reduced to the representation of object surfaces that it contains, without loosing their original smoothness. Several advantages are found in this representation: objects can be deformed or displaced by sub-voxel distances only by changing the coefficients (displacements greater than the voxel size will change also the voxels which belong to the smooth surface). Interactions between object surfaces can be performed in a more realistic way, (collisions, soft contacts). Interactions between object surfaces and light beams can avoid aliasing effects originated by the limited set of orientations of the voxels faces.

Figure 2. Mlustrates the object, the faces in its surface (top left). The smooth surface representation with coefficients that are positive on one side, and negatives on the other (top right). The smooth surface after a sub-voxel displacement (bottom left). The interaction between a ray (voxels with circles) and the smooth surface (bottom right). Surface normals are easily evaluated by using the coefficients of the voxels in the smooth surface representation.

To obtain the coefficients for the surface representation, a signal processing method is used. An edge detector operator based on geometric moments is applied to estimate the sub-voxel distance between the voxel center and the object boundary [12][13]. It retrieves positive values for the interior voxels and negative values for the exterior voxels.

\section{Line Tracing Method}

To cast a light beam, a discrete straight line should be traced. The line is defined by an origin point and a vector. The algorithm presented here is as simple and performant as the classical Bresenham's algorithm [11]. It should be noted that a light beam is a connected set of voxels, therefore it is a three dimensional object and not a one-dimensional manifold.

The first step is to realize a partition of all the possible directions in the grid (which corresponds, in the Bresenham's algorithm, to finding the octant in which lies the line). Here, the valid directions are those perpendicular to the faces of the Voronoi polyhedron. Figure 3 , shows three of them (vectors $\vec{A}, \vec{B}, \vec{C}$ in the two Voronoi cells. These vectors are grouped in sets of three elements. Vectors in the same set correspond to faces of the Voronoi polyhedron having a vertex in common. The linear combinations of this three vector spans a triangular pyramid with its vertex in the polyhedron center.

Figure 3. Illustrate the geometric configuration for the two Voronoi cells. Vector $\{\vec{A}, \vec{B}, \vec{C}\}$ are normal to the voxel faces with the common vertex $V$. The region defined by the points $\{a, e, b, f, c, d\}$ is the intersection of the pyramid spanned by vectors $\{\vec{A}, \vec{B}, \vec{C}\}$ with the polyhedron surface. Any grid vector originated in the polyhedron center and passing through this region can be ex- 


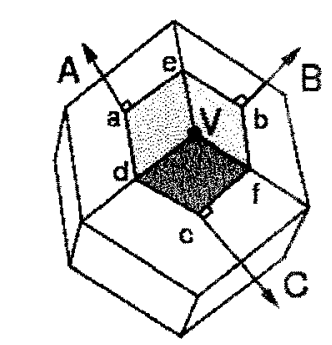

Rhombic Dodecahedron (FCC)

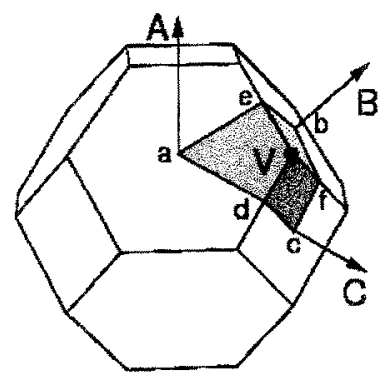

Truncated Octahedron (BCC)

Fig. 3. Partition of directions

pressed as a linear combination the vectors $\{\vec{A}, \vec{B}, \vec{C}\}$ with integer positive coefficients. There are 24 of these regions in the BCC, and 20 in the FCC grid.

The components $\left\{n_{a}, n_{b}, n_{c}\right\}$ of the line vector $P Q$ in the basis $\{\vec{A}, \vec{B}, \vec{C}\}$, means geometrically that to go from $P$ to $Q, n_{a}$ steps must be performed in $A$ direction, $n_{b}$ in $B$ direction and $n_{e}$ in $C$ direction. Figure 4 (left), represents the line tracing process from point $P$ to point $Q . N$ is the plane normal to the line. $R$ is the rhombic base of the prism which contains all the line beam points. The algorithm minimize the area of the rhombus $R$. Only integer sums should be computed to advance from one point to the next. Figure 4 (right) shows the projection of the grid points on the plane orthogonal to $P Q$, it is composed of a set of $2-D$ grids shifted by a constant vector.
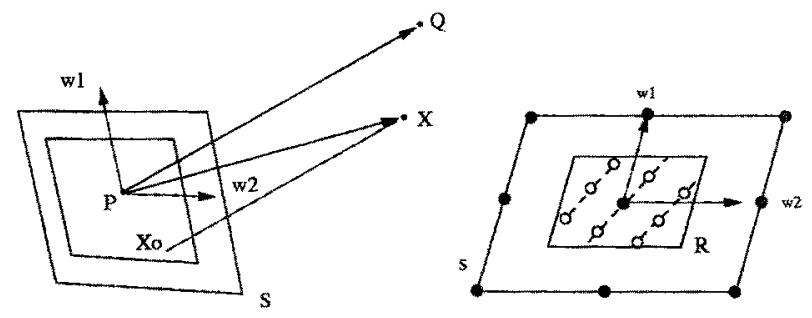

Fig. 4. Ray casting scheme

\section{Results}

The object representation method, and the ray casting algorithm described here, is used as a ray casting engine in a direct volume rendering program. The BCC grid is used as support of the volume data. Figure 5 shows the rendering of 

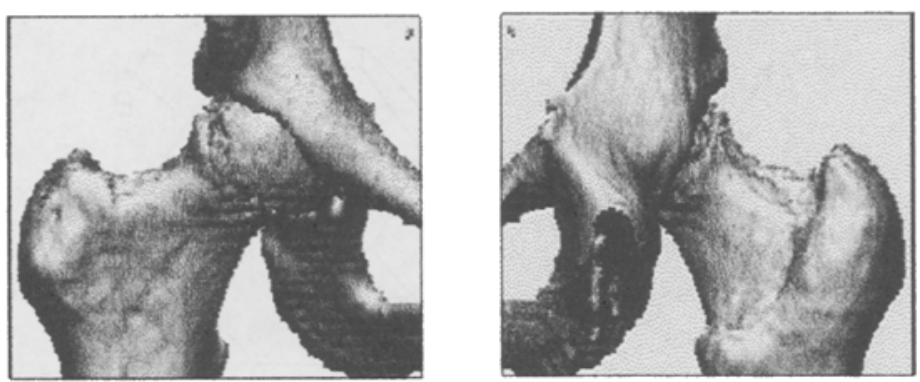

Fig. 5. Medical data volume in BCC grid support (Hip Joint)

a medical image using this program. The algorithm is implemented in $\mathrm{C}++$, on a $\mathrm{PC}$-Pentium $166 \mathrm{MHz}$ platform, running Linux 2.0 .18 . The original volume corresponds to a CT reconstruction of the hip joint containing $100 \times 100 \times 100$ voxels. It has been resampled using a $3 \mathrm{D}$ cubic B-Spline interpolator, to obtain a BCC structure. This resampling process duplicate the number of voxels. Output image has $256 \times 256$ pixels. Rendering time is $3 \mathrm{~s}$, it includes ray tracing and lighting calculus with one light source, with antialiasing.

\section{Conclusions}

The methods described in this article performs ray casting in volume data sampled with the BCC and FCC grids. The object representation is complete enough to allow process as object to object interaction, and object to light interaction to be done. The properties of these grids simplify the process involved in ray tracing and object description. It contributes to show that these grids can be used for the same purposes as the cubic grid, with the advantage of reduced number of samples and a well defined topology.

Algorithm performance is good enough to show that the choice of this topology does not imply an overload in rendering calculus with respecto to the cubic grid.

\section{References}

1. D.E.Dudgeon, R.M. Mersereau Multidimensional digital signal processing PrenticeHall, Englwood Cliffs; NJ; 1984.

2. C.Kittel Introduction to solid-state physics John Wiley \& Sons Inc. 1971.

3. J.Serra Image Analysis and Mathematical Morphology. Academic Press Inc. 1982.

4. Arie E. Kaufman, Volume Symthesis 6th International Workshop, Discrete Geometry for Computer Imagery 96, Lyon, France, November 1996. Lecture Notes in Computer Science, Springer Verlag. 
5. J.J.Jacq, C.Rowx, A Direct Multi-Volume Rendering Method Aiming at Comparisions of 3-D Images and Models IEEE Transactions on information technology in biomedicine, Vol 1., No.1, pp 30-43, march 1997.

6. G.T.Herman, 3D Display: A Survey From Theory to Applications Computerized Medical Imaging and Graphics, Vol. 17, Nos 4/5, pp 231-242, 1993.

7. W.Krüger, P.Schröder. Data parallel volume rendering pp 37-52. in Scientific Visualization, Academic Press, 1994.

8. U.Tiede, et al, Investigation of Medical $3 D$-Rendering Algorithms IEEE Computer Graphics \& Applications, Vol. 10, No.2, pp 41-53, 1990.

9. G.Sakas, M.Grimm, A.Savapoulos Optimized Maximum Intensity Projection (MIP). in Rendering Techniques'95, Proceedings of the Eurographics Workshop in Dublin, Ireland, June 12-14, Springer Verlag, 1995.

10. S. Matej, R.M. Lewit Efficient 3-D Grids for Image Reconstruction Using Spherically Symmetric Volume Elements IEEE Transactions on Nuclear Science, Vol 42, No.4, August 1995, pp 1361-1370.

11. J.E. Bresenham Algorithm for computer control of a digital plotter IBM Systems Journal, 1965, Vol 4. pp 25-30.

12. Li Min Luo, Ch. Hamitouche, L. Dillenseger, J.L. Coatrieux A Moment-Based Three-Dimensional Edge Operator, IEEE Transactions on Biomedical Engineering,. Vol 40, No.7, Jul 1993. pp 693-703.

13. L. Ibáñez, C. Hamitouche, C. Roux Moment-based operator for sub-voxel surface extraction in medical imaging International Conference on Image Processing ICIP'96, Lausanne, Switzerland, September 1996.

14. L. Ibáñez, C. Hamitouche, C. Roux Determination of discrete sampling grids with optimal topological and spectral properties 6th International Workshop, Discrete Geometry for Computer Imagery 96, Lyon, France, November 1996. Lecture Notes in Computer Science, Springer Verlag. 